STUDI

FRANCESI

\section{Studi Francesi}

Rivista quadrimestrale fondata da Franco Simone

190 (LXIV | I) | 2020

Varia - fasc. I - gennaio-aprile 2020

\title{
FERROUDJA ALLOUACHE, Archéologie du texte littéraire dit «francophone» 1921-1970
}

\section{Alexandre Calvanese}

\section{(2) OpenEdition}

\section{Journals}

\section{Édition électronique}

URL : https://journals.openedition.org/studifrancesi/23284

DOI : 10.4000/studifrancesi.23284

ISSN : 2427-5856

\section{Éditeur}

Rosenberg \& Sellier

\section{Édition imprimée}

Date de publication : 1 avril 2020

Pagination : 222-224

ISSN : 0039-2944

\section{Référence électronique}

Alexandre Calvanese, « FERroudja AllouAche, Archéologie du texte littéraire dit «francophone» 1927-1970 », Studi Francesi [En ligne], 190 (LXIV | I) | 2020, mis en ligne le 01 mai 2020, consulté le 03 août 2021. URL : http://journals.openedition.org/studifrancesi/23284 ; DOI : https://doi.org/10.4000/ studifrancesi.23284

Ce document a été généré automatiquement le 3 août 2021

\section{(c) (i) (9)}

Studi Francesi è distribuita con Licenza Creative Commons Attribuzione - Non commerciale - Non opere derivate 4.0 Internazionale. 


\title{
FERROUDJA ALLOUACHE, Archéologie du texte littéraire dit "francophone» 1921-1970
}

\author{
Alexandre Calvanese
}

\section{RÉFÉRENCE}

FERROUDJA ALLOUACHE, Archéologie du texte littéraire dit «francophone» 1921-1970, Paris, Classiques Garnier, 2018, 458 pp.

1 Ferroudja Allouache, maître de conférences à l'université Paris 8 - Vincennes - SaintDenis, publie chez les Classiques Garnier une œuvre qui mérite sans aucun doute l'attention de tout lecteur intéressé aux littératures que l'on appelle communément «francophones» et à la dynamique complexe de leur réception par la critique française. Les enjeux de cette étude - qui présente au public les résultats d'une recherche approfondie et richement documentée - sont de taille: selon les mots de l'auteure, il s'agit «de reconstituer la lente histoire de l'inscription des textes francophones dans le champ littéraire français et d'analyser la fabrication du concept "francophonie" qui a émergé au début des années 1960 et a désigné par la suite les écrivains des anciennes colonies françaises» (p. 7). Le but final est l'élaboration d'une contre-histoire littéraire francophone ou, si l'on préfère, le dévoilement d'une histoire «oubliée ou occultée, invisible ou peu prise en compte dans le champ littéraire de l'époque coloniale et sur une courte période post-coloniale» (ibidem).

2 Pour ce faire Allouache a consulté, recensé et analysé un corpus formé de dix-sept revues, quinze quotidiens et suppléments littéraires sans compter d'autres textes critiques (préfaces, notes de lecture, monographies), tous publiés entre les deux bornes temporelles indiquées dans le titre (qui correspondent respectivement à la sortie du roman Batouala de René Maran et à la publication chez Seuil de Les Soleils des indépendances d'Ahmadou Kourouma). Elle a passé ce corpus au crible pour en extraire 
les jugements - mais aussi les silences, souvent bien plus éloquents et significatifs - qui ont accompagné la parution des œuvres des écrivains issus des pays colonisés. Ce travail de longue haleine a ainsi permis «de débusquer les traces discursives qui tendent à marginaliser un auteur, ou au contraire à le faire entrer dans le panthéon littéraire» (p. 9), et de mettre en lumière le fait qu'un élément d' «inclusion» (le partage de la langue française) ne suffit pas à conjurer le risque d' "exclusion» d'un texte (puisque son origine est extra-nationale). En effet, le problème rencontré par les textes dits francophones a toujours été celui d'être (ou de ne pas être) reconnus et (dé)légitimés par le centre, tandis que pour ce dernier (c'est-à-dire la France) le problème a toujours été, dès l'apparition des premières œuvres de ces «nouveaux venus» issus des colonies, celui de trouver une stratégie pour se protéger contre des fictions «porteuses d'une histoire non audible» (p. 8), contre le péril de «toute intrusion possiblement déstabilisante» (p. 14).

3 C'est en relevant cette dynamique bien réelle - et donc pas à partir d'une réflexion abstraite - que pour Allouache s'impose l'exigence de reconsidérer le concept de francophonie car, observe-t-elle, si «le texte littéraire "francophone" ne posait pas problème, la différence entre ce qui relève de l'espace francophone et ce qui est français/hexagonal» ne tiendrait plus (p. 27), et le malentendu véhiculé par cet adjectif (que le monde français de l'édition et de la critique utilise comme synonyme de «tout ce qui n'est pas français») n'aurait plus cours. Souligner avec des guillemets - comme dans le titre de ce livre - le terme «francophone», est donc une manière d'en refuser tout emploi trop naïf, de «déconstruire l'impensé qui travaille le discours de la critique centrale» autour de la notion de francophonie (p. 9), de rappeler que cette même notion (qui était utilisée au départ dans un but exclusivement politique mais a glissé insensiblement vers le domaine de la littérature) demeure problématique sinon ambiguë, et encore de ne pas oublier que, si d'un côté «[s]ous cette terminologie se retrouvent ainsi classés ceux et celles dont le français n'est pas un héritage lié au prestige cosmopolite mais à un rapport de domination et d'imposition d'une langue» (p. 34), de l'autre côté le concept a «fabriqué une communauté littéraire» pour ceux qui n'en avaient pas (p. 426).

4 La démarche d'Allouache, qui se propose de trouver des traces dans les archives, se veut donc empirique, mais en même temps elle s'appuie sur de solides bases théoriques: les concepts foucaultiens d'archéologie et de généalogie, les réflexions de Michel de Certeau sur les marges et sur les dominés qui s'efforcent de gagner un interstice de manœuvre à l'intérieur de l'espace dominant, et - dans le sillage d'un ouvrage comme Les champs littéraires africains de Fonkoua et Halen (Karthala, 2001) - la notion de champ de Pierre Bourdieu. Cette théorie met en évidence la double invalidation dont souffrent les écrivains dominés: exclus du champ du pouvoir (politique et économique), leur place à l'intérieur du champ littéraire est souvent accordée au détriment de la dimension véritablement esthétique de leurs œuvres, que la critique continue de recevoir comme des documents historiques ou des témoignages d'engagement politique plutôt que comme des textes littéraires.

5 Pour donner un aperçu de cet ouvrage extrêmement riche et complexe (en raison des problématiques abordées et de l'ampleur du corpus mobilisé), on dira qu'il s'articule en trois parties, chacune recouvrant trois grandes périodes: 1921-1946, 1947-1961, 1962-1970. Ce découpage met en évidence, dans l'histoire de la réception des œuvres francophones, une succession de phases caractérisées par des traits communs. Pour 
chaque partie, Allouache propose des enquêtes (sept au total) sur des figures d'auteurs dont le parcours représente un éclairage singulier de la dynamique d'attraction/ répulsion ou inclusion/exclusion qui marque la relation entre le centre français et ses marges. Dans la première partie («Le cadre historique», pp. 21-162), Allouache décrit le fonctionnement de ce qu'elle appelle la «fabrique de l'invisibilité». La période prise en examen (1921-1946) est en effet celle où des revues françaises de premier plan telles que la "Revue des deux mondes" et "La Nouvelle Revue française" "se distinguent des autres par l'absence de toute référence aux textes d'écrivains en situation de colonisés» (p. 44), tandis qu'en même temps elles réservent une place d'honneur aux auteurs européens de langue française (comme Irène Némirovsky). D’autres revues, comme "Les Cahiers du Sud" et "Esprit", tout en accueillant et donnant visibilité à des auteurs comme Senghor, Damas, Amrouche, n'ont pas toujours échappé au piège d'approcher les textes davantage pour leur contenu documentaire (historique ou anthropologique) que pour leur valeur esthétique.

6 L'analyse des préfaces fait ressortir deux tendances opposées: une attitude, pour reprendre les mots de François Noudelmann, de "paternité sans fraternité», qu'Allouache identifie par exemple dans la posture d'un écrivain (de gauche) comme Jean-Richard Bloch, dont la préface à Force-Bonté du Sénégalais Bakary Diallo révèle «la condescendance, le jugement de classe et une perception racialisée» (p. 63); et une attitude, en rupture avec la précédente, de «fraternité sans paternité» qu'Allouache retrouve dans la préface signée par le poète surréaliste Desnos au recueil de poèmes Pigments du poète guyanais Léon Gontran Damas. Les deux figures censées illustrer cette période sont le romancier guyanais René Maran et le poète berbère-chrétien Jean ElMouhouv Amrouche. Le premier est le seul auteur dominé qui ait rédigé lui-même une préface pour son roman (Batouala), et Allouache se demande si la réception peu bienveillante de son œuvre de la part de la critique française ne serait pas une réponse à cet «acte inattendu» d'insoumission et d'affranchissement (p. 97). Le deuxième incarne un paradoxe déchirant: il réclame une ascendance culturelle et littéraire française et européenne en rupture avec son milieu socio-culturel d'origine, il arrive même à fréquenter l'élite littéraire de son temps (il se liera d'amitié avec Gide) avant de découvrir l'hostilité que ce monde lui réserve, et qui l'empêchera de devenir ce qu'il espérait, c'est-à-dire «un parfait assimilé».

7 La deuxième partie, «Présence et absence. Une production littéraire existante mais invisible» (pp. 163-301), se focalise sur une période cruciale, aussi bien d'un point de vue historico-politique que littéraire. Les mouvements anticolonialistes et tiersmondistes montent en puissance (rappelons que pour la plupart des colonies françaises en Afrique le processus de décolonisation aboutira en 1960 avec l'indépendance), et ce contexte est propice à l'émergence de grandes figures intellectuelles afro-antillaises jusqu'à ce moment peu visibles (raison pour laquelle l'adjectif «invisible» dans le titre de cette partie est peut-être un peu déroutant). Allouache propose des analyses très perspicaces des moments capitaux qui marquent ces années. On retiendra celle qui est consacrée à la naissance de la revue Présence africaine, fondée à Paris par Alioune Diop en 1947. Allouache commence par rappeler que le jeu des alliances et les réseaux amicaux et intellectuels au sein du champ littéraire de l'époque (Diop bénéficia du soutient d'intellectuels comme Sartre, Camus, Leiris, Bataille) furent décisifs pour détourner la revue du destin éphémère de nombreuses autres publications du monde afro-antillais; elle dévoile ensuite la fine stratégie dialectique de Diop pour déjouer le discours dominant et s'installer dans l'espace littéraire institutionnel; elle relève enfin 
que Diop instaura à l'intérieur de sa revue le même «esprit de famille» qui caractérisait la "NRF", avec les mêmes rituels de sélection, d'introduction et de légitimation du nouvel entrant selon lesquels «les auteurs reconnus et légitimés commentent les nouveaux arrivants» (p. 203).

Un autre tournant analysé par Allouache est la publication, en 1948, de l'Anthologie de la nouvelle poésie nègre et malgache établie par Senghor. C'est probablement grâce à la préface de Sartre («Orphée Noir») que cette anthologie deviendra «la référence dans toutes les histoires littéraires écrites après les indépendances» (p. 180); comment expliquer autrement, s'interroge Allouache, le fait que seulement l'année précédente deux anthologies parues en France (Poètes d'expression française éditée par Damas et Les Plus beaux écrits de l'Union française et du Maghreb présentée par un collectif dont Senghor lui-même faisait partie) étaient passées presque inaperçues, et qu'ensuite elles sont restées pratiquement absentes «dans la mémoire des littératures francophones parues à partir des années 1980» (p. 179)? Allouache montre que les préfaces jouent en effet un rôle majeur dans cette phase, en particulier si leur auteur s'appelle Jean-Paul Sartre. Cet «intellectuel total» contribue à la consécration, outre celle de Senghor, d'Albert Memmi (dont Sartre préface le Portrait du colonisé, publié en 1957) et de Frantz Fanon (dont il préface Les Damnés de la terre, paru en 1961). C'est à ces trois intellectuels, et aux trois préfaces sartriennes qui reprennent «le discours du colonisé afin de théoriser les contradictions du système colonial» (p. 258), que sont consacrés les trois approfondissements de cette partie.

9 Dans la troisième partie («Réception et fabrique de l'a-généalogie», pp. 303-424), Allouache se focalise sur les conséquences que la décolonisation a eues sur la réception des textes des auteurs désormais décolonisés. Elle note une différence substantielle dans l'accueil réservé d'un côté aux écrivains francophones du Proche et Moyen-Orient, qui «sont toujours insérés dans l'histoire littéraire hexagonale» (p. 306), et de l'autre aux écrivains afro-antillais et maghrébins, toujours tenus à l'écart, sans filiation, et assignés à la nouvelle catégorie de "francophonie». Le contexte politique continue d'orienter la réception, qui se focalise principalement sur le caractère revendicatif des œuvres au détriment de leur dimension littéraire, rarement objet d'une analyse approfondie: "À défaut de pouvoir identifier les textes comme témoignage sur les indépendances ou comme récits sur la guerre et ses séquelles, les écrits qui rompent avec la signification immédiate, qui brouillent les frontières énonciatives, désorientent ou surprennent, n'ont pas de place dans le champ littéraire hexagonal. L'incapacité à accorder le statut d'auteur résiste » (p. 323). La première étude de cas de cette partie est par contre réservée à un geste qui subvertit cette tendance: la préface de Louis Aragon à Ombre gardienne de l'écrivain algérien Mohammed Dib marque en effet une rupture dans la réception hexagonale, car «l'écrivain n'est plus présenté inféodé à sa condition de colonisé, mais pour ce qu'il est: poète» (p. 330).

Allouache se penche ensuite sur les tentatives de définir la production littéraire des anciennes colonies et sur la manière de construire (ou d'ignorer) sa généalogie. À ce propos elle esquisse une analyse comparée d'abord des anthologies de Léonard Sainville (Anthologie de la littérature négro-africaine. Romanciers et conteurs, 1963) et d'Albert Memmi (Anthologie des écrivains maghrébins d'expression française, 1964), ensuite des travaux d'Auguste Viatte (Anthologie littéraire de l'Amérique française, 1954) et de Lylian Kesteloot (Les Écrivains noirs de langue française: naissance d'une littérature, 1963). Se tournant ensuite vers les revues, elle montre que l'idée de francophonie est presque 
absente des discours des intellectuels des anciennes colonies (avec l'exception de Senghor, qui la considère une redéfinition de l'Humanisme français), tandis qu'elle «semble devenir une préoccupation hexagonale puisque critiques et chroniqueurs y consacrent de nombreux articles», et que le concept «devient une manière de rapatrier au centre, de nationaliser ce qui n'appartient plus à l'ancien Empire» (p. 356). En outre, l'emploi du terme a presque toujours un sens exclusivement politique, renvoyant à une idée d'assistance envers les pays africains.

11 Enfin, Allouache aborde la question de la langue qui «fabrique le littéraire», car «[e]n cette période de transition, il n'est pas encore évident, pour un écrivain, d'affirmer sa liberté totale à l'égard d'une langue dont il se sert [...] mais qui lui rappelle, sans cesse, qu'elle est intimement liée à une histoire non cautérisée» (p. 382). La dernière figure de l'ouvrage ne peut être alors qu'Ahmadou Kourouma qui, avec Les Soleils des indépendances, incarne «la possibilité d'un enrichissement de la langue française par un auteur que le centre peine à reconnaître» (p. 422). Le parcours éditorial de son roman reste en effet emblématique: refusé par les maisons d'édition françaises en raison aussi de ses inventions linguistiques, il est «accueilli et [...] récompensé par la marge» (à savoir l'Université de Montréal et la revue canadienne "Études françaises") avant d'être récupéré par le centre (les éditions du Seuil, qui rachètent les droits pour un franc symbolique).

12 En conclusion, on ne peut que louer le travail de Ferroudja Allouache, et lui pronostiquer un avenir comme texte de référence dans le domaine des études sur les littératures dites «francophones». 